\title{
DRL performance in rats following damage to the septal area, olfactory bulbs, or olfactory tubercle
}

\author{
B. MICHAEL THORNE, KEITH RAGER, and JEFF S. TOPPING \\ Mississippi State University, Mississippi State, Mississippi 39762
}

\begin{abstract}
Long-Evans rats with olfactory bulb damage, olfactory tubercle destruction, septal lesions, or control operations were trained on a VI 30 -sec schedule and tested for response suppression ability on DRL 10-sec and DRL 20-sec schedules. Animals with septal damage overresponded on all three schedules, while animals with olfactory bulb damage overresponded on the VI 30 -sec schedule and on the DRL 10-sec schedule. Control subjects and animals with olfactory tubercle damage did not differ on any measure. A functional relationship between the septal area and the olfactory bulbs is probably not mediated by pathways through the olfactory tubercle.
\end{abstract}

Many of the behavioral effects seen in rats following removal of the olfactory bulbs (OB) are quite similar to effects seen after damage to the septal area. For example, increased barpressing has been reported after olfactory bulb damage (Marks, Remley, Seago, \& Hastings, 1971; Sieck, 1973; Thorne, McDougal, \& Topping, 1976) and after septal destruction (Aaron \& Thorne, 1975; Ellen \& Powell, 1962; Harvey \& Hunt, 1965; Hothersall, Johnson, \& Collen, 1970). Additionally, deficits have been seen on tasks requiring the ability to suppress a previously reinforced response in both septally damaged (Aaron \& Thorne, 1975; Burkett \& Bunnell, 1966; Winocur \& Mills, 1969) and bulbectomized rats (Brown, Harrell, \& Remley, 1971; Sieck, 1973; Thorne et al., 1976).

In a recent study in which an attempt was made to compare rats with $\mathrm{OB}$ damage with rats from a previous study in which the septal area was damaged (Thorne et al., 1976), the proposal was made that because of the striking quantitative similarities in behavior, the OB and septum may be part of a functional system mediating response inhibition. Additional evidence for the functional relatedness of the areas comes from several recent studies demonstrating changes in olfactory sensitivity after septal damage (Carlson \& Vallante, 1974; Kemble \& Nagel, 1975; Vom Saal, Hamilton, \& Gandelman, 1975). For example, Vom Saal et al. found that rats with septal damage acquired an olfactory discrimination more rapidly than normals. The authors hypothesized an inhibitory influence of the septal area on the bulbs via interconnections in the olfactory tubercle.

As a test of the hypothesis that the septal area and the olfactory bulbs are part of a functional system mediating response inhibition, the present study assessed the effect of olfactory tubercle damage on response suppression in rats. If the septal area exercises an inhibitory effect upon the olfactory bulbs via pathways within the olfactory tubercle, damaging these pathways should have an effect upon response suppression ability similar to that seen after septal or olfactory bulb damage, i.e., overresponding.

Since a different strain of rat was employed in the present study (Long-Evans) than had been used in previous reports from this laboratory (SpragueDawley, Aaron \& Thorne, 1975; Thorne et al., 1976), animals with either septal damage or OB removal were included to determine whether or not the previous results were strain-specific. To measure response suppression ability, all animals were tested on differential reinforcement of low rate paradigms (DRL 10-sec and DRL 20-sec schedules) following training on a variable interval schedule (VI 30-sec).

\section{METHOD}

\section{Subjects}

The subjects were 51 adult male hooded rats of the Long-Evans strain. All animals were obtained from the breeding stock maintained by the Psychology Department of Mississippi State University.

Because of either untrainability, postoperative death, or improper lesion placement, 15 rats were eliminated from the study, leaving 9 subjects in each of four treatment groups: Group Operated Control (OC), Group Olfactory Tubercle (OT), Group Olfactory Bulb (OB), Group Septal (SEP). All subjects were at least 90 days old at the time of surgery. The average preoperative weight of all rats completing the study was $488.1 \mathrm{~g}$.

All animals were kept in group cages from weaning until approximately 1 week prior to surgery, at which time they were placed in single cages measuring $17.78 \times 25.40 \times 17.78 \mathrm{~cm}$. Purina Lab Chow was available ad lib prior to surgery and for the first 4 postoperative days, while free access to water was permitted throughout the experiment.

\section{Surgery and Histology}

At the time of surgery, the animals were removed from their cages, weighed, and anesthetized with chloral hydrate solution. 
The animal's head was secured in the headholder from a Baltimore stereotaxic instrument with the top of the skull approximately horizontal with respect to the base of the headholder for rats in Groups OB and SEP. For animals in Group OT, the toothbar was elevated $5 \mathrm{~mm}$ above the earbars.

After exposure of the bulbs, removal was performed by subpial aspiration. Following appropriate exposure of the surface of the brain, damage to either the septal region or the olfactory tubercle was made by the electrolytic method. The coordinates used for damaging the septal area were as follows: $0 \mathrm{~mm}$ anterior with respect to bregma, $.5 \mathrm{~mm}$ lateral to the midsagittal sinus, $5-5.5 \mathrm{~mm}$ ventral to the pia mater. The current employed was 1-2 mA for $10-20 \mathrm{sec}$. Damage to the olfactory tubercle was effected by two electrode placements on each side of the midline. The coordinates used were $5 \mathrm{~mm}$ anterior, $1.5 \mathrm{~mm}$ lateral, $7.5 \mathrm{~mm}$ ventral, and $4 \mathrm{~mm}$ anterior, $2 \mathrm{~mm}$ lateral, and $8.5 \mathrm{~mm}$ ventral. The current employed was $1 \mathrm{~mA}$ for $10 \mathrm{sec}$. Control operations involved all of the operative procedure, except that neural tissue was not damaged.

After testing was completed, all operated animals were sacrificed with an overdose of Nembutal. The brains of animals in Groups OT and SEP were extracted and placed into $10 \%$ Formalin solution for at least 1 week prior to sectioning. Sections were made at 100 microns with a freezing microtome, placed on glass slides, and photographed after 10X enlargement.

For Group OB subjects, the tops of the skulls were removed and the entire head placed into $10 \%$ Formalin solution for at least a week. The brains were then extracted and the extent of damage was reconstructed on line drawings of the dorsal surface of a rat brain. From the line drawings, an estimate was made of the extent of damage rostral to the frontal poles, using the method described by Thorne, Aaron, and Latham (1973).

\section{Training and Testing}

All rats were allowed to recover from surgery for 7 days prior to initiation of training. After ad-lib food for the first 4 days, the animals were given a reduced ration once daily for the last 3 recovery days. In addition, a small dish containing several 45-mg Noyes pellets was placed in each animal's cage during the recovery period in order to hasten finding and eating of the pellets during shaping. All subjects were weighed daily beginning with the first training day and were given a food ration necessary to reduce and maintain their weight at approximately $80 \%$ of their preoperative weights.

On the 8th day following surgery, each subject was placed in a standard Lehigh Valley operant conditioning chamber and was trained to press the bar for $45-\mathrm{mg}$ Noyes pellets. Shaping was considered complete when the animal had received at least 20 response-contingent reinforcements within a single session. During shaping, the length of the sessions varied from a minimum of 20 min to a maximum of $35 \mathrm{~min}$.

After the animals had acquired the response, at least one session of adjustment to partial reinforcement was given. Adjustment consisted of training on a variable ratio schedule requiring a gradually increasing number of responses per reinforcement and was considered complete when the rat was consistently averaging at least 6 responses/reinforcement.

On the day after successful completion of the adjustment, all subjects were placed on a VI 30 -sec schedule and remained on this schedule until their response rate stabilized. The criterion of stabilization was defined as 3 consecutive days with less than $15 \%$ variability in the total number of responses. Responses on the first 4 days of VI 30 -sec training were not considered in deciding whether or not the response rate had stabilized so that the minimum number of days any animal stayed on the VI 30-sec schedule was 7. All sessions beginning with the introduction of VI training were $20 \mathrm{~min}$ in length.

On the day after stabilization on the VI 30-sec schedule, each animal was placed on a DRL 10 -sec schedule. On the DRL 10-sec schedule, any barpress occurring with less than a 10-sec delay from the previous press reset a timer and thus delayed reinforcement by at least $10 \mathrm{sec}$. A barpress following a previous press by $10 \mathrm{sec}$ or more resulted in reinforcement and reset the timer initiating another cycle. All subjects were given 7 days on the DRL 10-sec schedule and were then switched to a DRL 20-sec schedule for 7 days. The DRL 20 -sec schedule was identical to the DRL 10-sec schedule, except that the delay requirement was 20 instead of $10 \mathrm{sec}$.

\section{RESULTS}

\section{Histology}

There was very little variability in the amount of OB tissue destroyed rostral to the frontal area in Group OB animals. The average percentage destroyed was approximately $81 \%$, with a range from about $64 \%-91 \%$. Four animals sustained frontal damage, although in each case it was minor.

The destruction of the medial and lateral septal nuclei was almost complete in nearly every subject in Group SEP. Extraseptal damage included the corpus callosum (usually minor), cingulate cortex, anterior commissure, diagonal bands of Broca, the fornix, and columns of the fornix. Unstained sections from a typical lesion are shown in Figure 1.

All animals in Group OT sustained bilateral damage almost exclusively confined to the olfactory tubercle. In most cases, the lesions were not extensive and extended from approximately $5 \mathrm{~mm}$ anterior to about $2 \mathrm{~mm}$ anterior to bregma. Structures sustaining minor damage in addition to the olfactory tubercle included the anterior olfactory

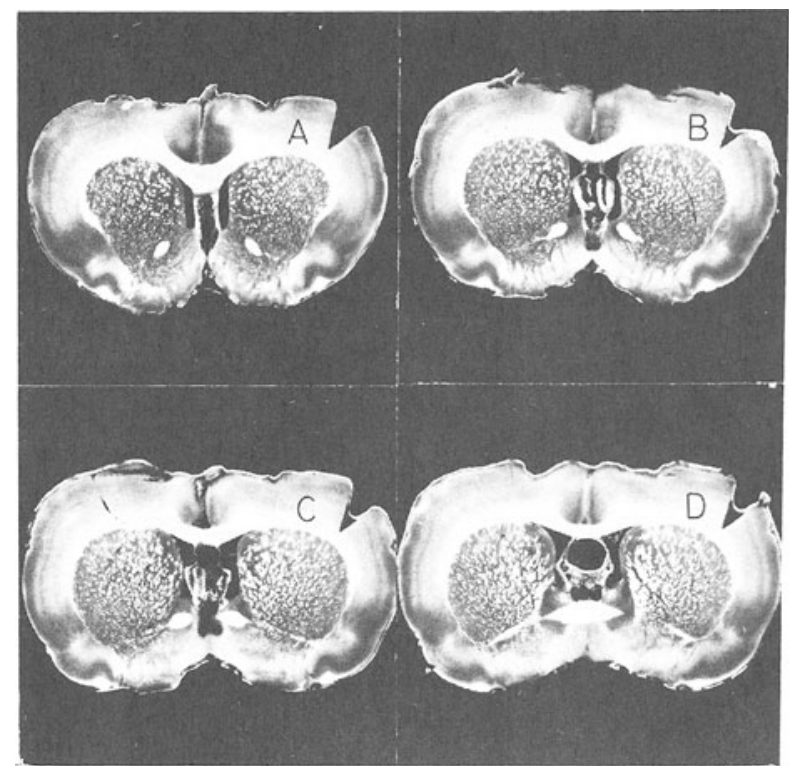

Figure 1. Unstained sections from the brain of an animal in Group SEP. Sections A-D depict septal damage at successive anterior-posterior levels of the brain. 


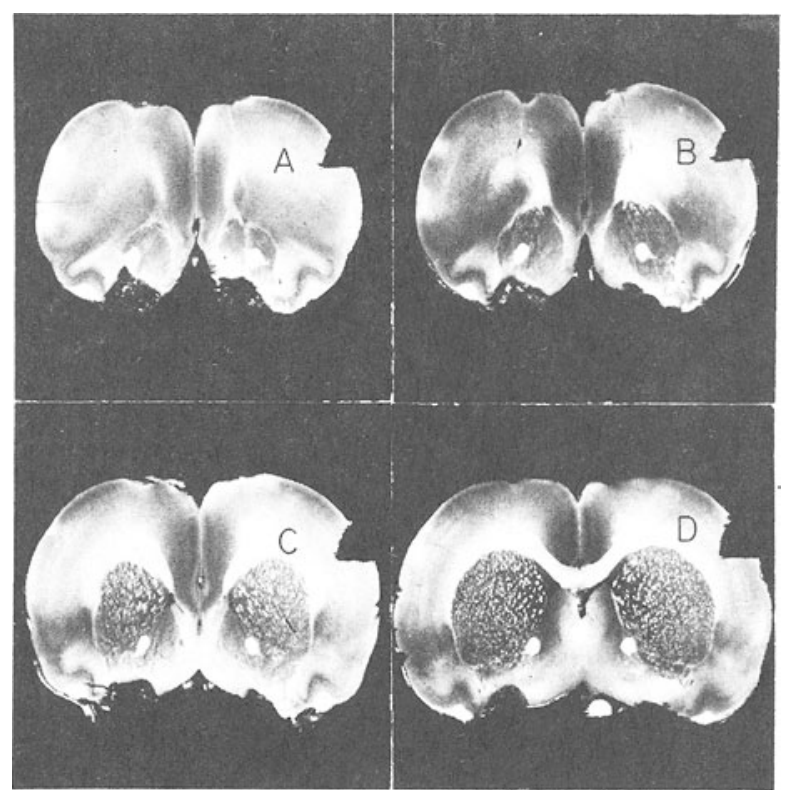

Figure 2. Unstained sections from the brain of an animal in Group OT. Sections A-D depict olfactory tubercle damage at successive anterior-posterior levels of the brain.

nucleus, medial forebrain bundle, nucleus accumbens septi, diagonal bands of Broca, and the lateral preoptic area. Unstained sections from a typical lesion are shown in Figure 2.

\section{30-sec Performance}

A one-way analysis of variance performed on the average number of responses per session on the last 3 days of VI 30-sec training revealed significant lesion effects, $F(3,32)=15.159, p<.001$. The group means were as follows: Group OC, 162.6; Group OT, 159.1; Group OB, 367.3; and Group SEP, 308.9. An a posteriori test (Newman-Keuls) indicated

Figure 3. Average number of reinforcements obtained each day during DRL 10 -sec training. that Groups OC and OT did not differ from each other but were significantly different from Groups OB and SEP, which did not differ. Thus, rats with septal or olfactory bulb damage barpressed at significantly higher rates than did animals with either control operations or olfactory tubercle damage. The rank order correlation between extent of damage and average number of responses per session was not significant for either Group OB or Group SEP animals.

\section{DRL 10-sec Performance}

A one-way analysis of variance performed on the total number of reinforcements obtained on the DRL 10 -sec schedule revealed significant group differences, $\mathrm{F}(3,32)=25.455, \mathrm{p}<.001$. Further analysis with the Newman-Keuls procedure indicated that Group SEP obtained significantly fewer reinforcements than Group OB, which was reinforced significantly less frequently than either Groups OC or OT. Groups $O C$ and OT did not differ. The daily mean number of pellets obtained by each subject within a group was: Group OC, 39.68; Group OT, 43.87; Group OB, 26.25; and Group SEP, 17.68. Extent of destruction in Groups $\mathrm{OB}$ and SEP was not related to DRL 10-sec performance.

The response rates during VI 30 -sec training were significantly related to number of reinforcements obtained on the DRL 10 -sec schedule for animals in all groups except Group OT $\left(\mathrm{r}_{\mathrm{s}}=-.68,-.57,-.72\right.$, and -.67 for Groups OC, OT, OB, and SEP, respectively). The correlation obtained for Group OT was very close to the value required for significance $\left(r_{\mathrm{s}}=-.60\right.$, one-tailed test $)$.

Figure 3 plots the development of DRL 10-sec performance as measured by the mean number of pellets per session across the 7 days of testing. Because the shape of the curves suggested that improvement across days might have occurred, a trend

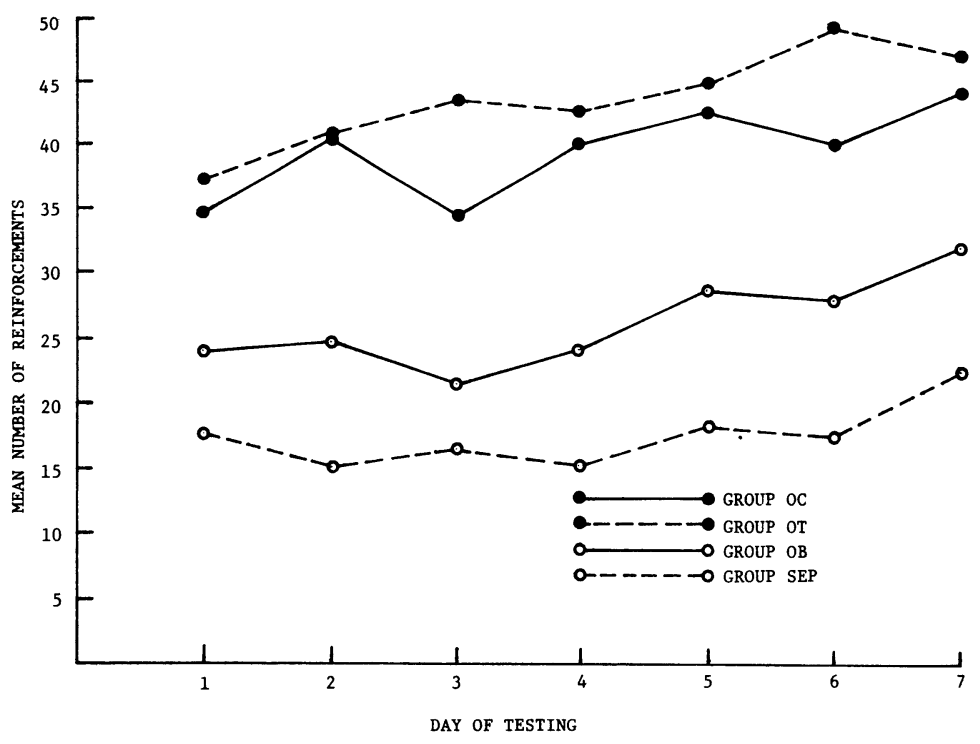




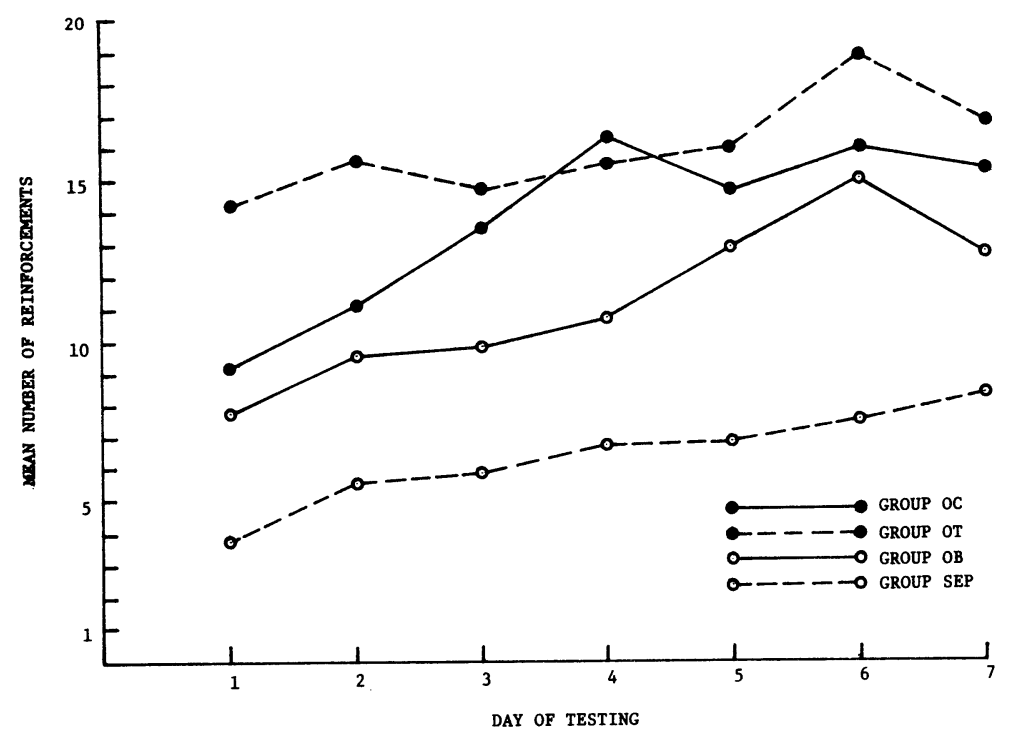

Figure 4. Average number of reinforcements obtained each day during DRL 20-sec training.

analysis was performed. All groups except Group SEP showed evidence for improvement across days: Group OC, $\mathrm{F}(6,48)=4.59, \mathrm{p}<.01$; Group OT, $F(6,48)=4.86, p<.01$; and Group OB, $\mathrm{F}(6,48)=3.18, \mathrm{p}<.05$.

\section{DRL 20-sec Performance}

A comparison of the total number of pellets obtained during DRL 20-sec training revealed significant group differences, $F(3,32)=14.91, \mathrm{p}<.001$. A posteriori comparisons using the Newman-Keuls procedure indicated that Groups OT and OC did not differ, Groups OB and OC did not differ, Group OB received significantly fewer pellets than did Group OT, and Group SEP obtained fewer pellets than all other groups. The daily mean number of pellets obtained by each animal within a group was: Group OC, 13.84; Group OT, 16.05; Group OB, 11.27; and Group SEP, 6.41.

The rate of responding during VI 30-sec training was significantly correlated with DRL 20 -sec performance for animals in Group OC $\left(r_{s}=-.90\right.$, $\mathrm{p}<.01$, one-tailed test) and approached significance for Group $\mathrm{OB}$ animals $\left(\mathrm{r}_{\mathrm{s}}=-.57,-.60\right.$ needed for significance).

The development of DRL 20-sec performance (as measured by the mean number of pellets per session) across the 7 days of testing is shown in Figure 4. Again, the curves suggest that improvement in performance occurred. Trend analyses revealed that the improvement was significant for all groups except Group OT: Group OC, $\mathrm{F}(6,48)=9.46, \mathrm{p}<.01$; Group OB, $F(6,48)=5.15, p<.01$; and Group SEP, $\mathrm{F}(6,48)=2.48, \mathrm{p}<.05$.

\section{Informal Behavioral Observations}

The animals in Groups OC and OT were very easy to handle postoperatively. For the most part, this was also true for Group OB rats with only two of nine animals exhibiting hyperirritability. In one of the two, the irritability to handling continued throughout the study, while the other was indistinguishable from a Group OC animal within a few days. Finally, five of nine animals in Group SEP were very difficult to handle for 7-14 days postoperatively, exhibiting biting, squealing, and frequent escape attempts.

\section{DISCUSSION}

The results of the present study replicated several previous reports (e.g., Aaron \& Thorne, 1975; Marks et al., 1971; Thorne et al., 1976) in finding that rats with septal damage or with olfactory bulb destruction responded at high rates on a barpressing task for food reinforcement relative to control subjects. In Thorne et al. (1976), a comparison was made between rats with septal damage and rats with $\mathrm{OB}$ destruction in terms of average barpressing rates on a VI $30-\mathrm{sec}$ schedule. When the average scores were expressed as multiples of control rates, both septally damaged rats and animals with $\mathrm{OB}$ destruction pressed at almost twice the control rate $(1.8$ and 1.9 , respectively). Applying a similar analysis to the animals in this study, we found that septally damaged rats pressed at 1.9 times the control rate while animals in Group $\mathrm{OB}$ averaged 2.3 times as many responses. Again, Group OB and SEP rats were not found to differ on this measure and responded at approximately twice the rate of control animals. It is obvious that the results from previous studies in this laboratory were not strain specific.

As in many previous studies (e.g., Aaron \& Thorne, 1975; Sieck, 1973; Thorne et al., 1976), rats with OB damage or with septal lesions were found to be deficient in the ability to suppress responding for appetitive reward. In the present study, rats with 
OB damage were worse than controls on the DRL 10 -sec task but did not differ significantly on the DRL 20-sec schedule, although Group OB animals averaged fewer pellets on each day of testing. Group SEP animals, on the other hand, received fewer pellets than either the controls or Group OB animals throughout DRL training.

Although Group OB rats were somewhat deficient on the DRL task relative to control animals, the deficit was not nearly as great as we had observed in a previous experiment (Thorne et al., 1976). An obvious explanation for this discrepancy is that the lesions were less extensive in the present study. Thus, in this experiment, the average amount of damage to the olfactory bulbs rostral to the frontal area was approximately $81 \%$, while it was about $94 \%$ in the previous report. Several studies have shown a correspondence between the extent of damage to the olfactory system and behavioral changes (e.g., Cain, 1974; Sieck \& Gordon, 1972; Thorne, Aaron, \& Latham, 1974).

The present study was designed to test the hypothesis that the septal area and the olfactory bulbs are functionally related in the control of response suppression ability by means of pathways through the olfactory tubercle. We found no evidence to support this hypothesis as animals in Group OT did not differ on any measure from Group OC animals. Additionally, Group OT rats were significantly different from Group $O B$ and Group SEP rats on all measures.

Although the evidence from the present report suggests that response suppression ability is unaffected by damage to pathways between the septal area and the $O B$ through the olfactory tubercle, the possibility of functional connections by means of other pathways is not eliminated. Thus, it has been demonstrated that the olfactory bulb projects to the pyriform cortex which in turn sends efferent fibers to the nucleus of the diagonal band of Broca (Powell, Cowan, \& Raisman, 1965). The nucleus of the diagonal band of Broca is in continuity with the medial septal area dorsally and both projects to and receives projections from the septum (Knook, 1965). One subject whose data were not included in this study because of asymmetry of damage and because no relevant structures were damaged (i.e., OT, OB, SEP), performed well within the range of Group OB and SEP subjects on all measures. The diagonal band of Broca was damaged bilaterally and extensively in this animal.

\section{REFERENCES}

Aaron, M., \& Thorne, B. M. Omission training and extinction in rats with septal damage. Physiology and Behavior, 1975, 15, 149-154.
Brown, G. E., Harrell, E., \& Remley, N. R. Passive avoidance in septal and anosmic rats using quinine as the aversive stimulus. Physiology and Behavior, 1971, 6, 543-546.

Burkett, E. E., \& Bunnell, B. N. Septal lesions and the retention of DRL performance in the rat. Journal of Comparative and Physiological Psychology, 1966, 62, 468-471.

CAIN, D. P. Olfactory bulbectomy: Neural structures involved in irritability and aggression in the male rat. Journal of Comparative and Physiological Psychology, 1974, 86, 213-220.

Carlson, N. R., \& Vallante, M. A. Enhanced cue function of olfactory stimulation in mice with septal lesions. Journal of Comparative and Physiological Psychology, 1974, 87, 237-248.

Ellen, P., \& Powell, E. W. Effects of septal lesion on behavior generated by positive reinforcement. Experimental Neurology, 1962, 6, 1-11.

HARVEY, J. A., \& Hunt, H. F. Effect of septal lesions on thirst in the rat as indicated by water consumption and operant responding for water reward. Journal of Comparative and Physiological Psychology, 1965, 59, 49-56.

Hothersall, D., Johnson, D. A., \& Collen, A. Fixed-ratio responding following septal lesions in the rat. Journal of Comparative and Physiological Psychology, 1970, 73, 470-476.

Kemble, E. D., \& NAGEL, J. A. Decreased sniffing behavior in rats following septal lesions. Bulletin of the Psychonomic Society, 1975, 5, 309-310.

KNook, H. L. The fibre-connections of the forebrain. The Netherlands: Van Gorcum, 1965.

Marks, H. E., Remley, N. R., Seago, J. D., \& Hastings, D. W. Effects of bilateral lesions of the olfactory bulbs of rats on measures of learning and motivation. Physiology and Behavior, 1971, 7, 1-6.

Powell, T. P. S., Cowan, W. M., \& Raisman, C. The central olfactory connexions. Journal of Anatomy, 1965, 99, 791-813.

SIECK, M. H. Selective olfactory system lesions in rats and changes in appetitive and aversive behavior. Physiology and Behavior, 1973, 10, 731-739.

SiEcK, M. H., \& Gordon, B. L. Selective olfactory bulb lesions: Reactivity changes and avoidance learning in rats. Physiology and Behavior, 1972, 9, 545-552.

Thorne, B. M., Aaron, M., \& LATham, E. E. Effects of olfactory bulb ablation upon emotionality and muricidal behavior in four rat strains. Journal of Comparative and Physiological Psychology, 1973, 84, 339-344.

Thorne, B. M., Aaron, M., \& Latham, E. E. Olfactory system damage in rats and emotional, muricidal, and rat pup killing behavior. Physiological Psychology, 1974, 2, 157-163.

ThORNe, B. M., McDougal, Y., \& TopPING, J. S. Olfactory bulb removal and response suppression in rats. Physiology and Behavior, 1976, 17, 259-265.

Vom SaAl, F. S., Hamilton, L. W., \& Gandelman, R. J. Faster acquisition of an olfactory discrimination following septal lesions in male albino rats. Physiology and Behavior, 1975, 14, 697-703.

Winocur, G., \& Mills, J. A. Hippocampus and septum in response inhibition. Journal of Comparative and Physiological Psychology, 1969, 67, 352-357.

\section{NOTE}

1. This research was in part supported by an institutional grant awarded to Mississippi State University by NSF. Address reprint requests to: B. Michael Thorne, P. O. Drawer PF, Mississippi State, Mississippi 39762.

(Received for publication August 9, 1976; accepted August 27, 1976.) 